\title{
Patient considerations in the management of gout and role of combination treatment with lesinurad
}

\section{Liza W Claus \\ Joseph J Saseen}

Department of Clinical Pharmacy, Skaggs School of Pharmacy and

Pharmaceutical Sciences, University of Colorado, Aurora, CO, USA
Correspondence: Liza W Claus Department of Clinical Pharmacy, Skaggs School of Pharmacy and Pharmaceutical Sciences, University of Colorado, Anschutz Medical Campus, I2850 E. Montview Boulevard, C238, Aurora, CO 80045, USA

Tel +l 3037249548

Email liza.claus@ucdenver.edu
This article was published in the following Dove Press journal: Patient Related Outcome Measures

\begin{abstract}
Gouty arthritis is one of the most common rheumatic diseases, and the prevalence continues to rise, which is likely related to increased incidence of comorbidities, lifestyle factors, and suboptimal utilization of urate-lowering therapy. In recent years, multiple new guidelines have been published along with the approval of novel drug therapies. Still, gout remains a poorly controlled disease state that is accompanied by a reduced health-related quality of life, increased health care utilization, and overall negative socioeconomic effects, all of which have a negative impact on patient-related health outcomes. The key to success in gout management is utilization of urate-lowering therapy to prevent recurrence of acute gouty arthritis and to resolve tophi, if present. Xanthine oxidase inhibitors are first-line medications for the prevention of recurrent gout followed by uricosurics, including lesinurad (a uric acid reabsorption inhibitor) as an add-on option. The recent US Food and Drug Administration Safety Communication related to cardiovascular risk with febuxostat may result in increased use of allopurinol in combination therapy with a uricosuric agent such as lesinurad. In this review, we discuss gout management, clinical end points, and patient-related outcomes for consideration, summarize the evidence for combination therapy to achieve serum urate targets, and focus on lesinurad as a novel newer medication for the prevention of gout.
\end{abstract}

Keywords: gouty arthritis, patient-centered, treatment, lesinurad

\section{Introduction}

Gout is the most common inflammatory arthritis among adults in the USA, and its prevalence is increasing in Western countries. Gout is a broad term used to define a spectrum of disease related to excess uric acid secondary to either overproduction or under excretion of serum urate. Persistently elevated uric acid can lead to gouty arthritis, deposition of palpable urate crystals in joints known as tophi, interstitial nephropathy, uric acid nephrolithiasis, and chronic kidney disease (CKD). Elevated uric acid is generally recognized as serum urate of $>7 \mathrm{mg} / \mathrm{dL}$. ${ }^{1}$ The serum urate goal of therapy for patients diagnosed with gout is $<6 \mathrm{mg} / \mathrm{dL}$ with an optional $<5 \mathrm{mg} / \mathrm{dL}$ in some patients ${ }^{1,2}$ and often requires the use of urate-lowering therapy (ULT). However, because acute gouty arthritis may rarely persist despite normal uric acid levels, therapy might also be guided with the goal of avoiding acute gouty arthritis (treat-to-avoid symptoms). ${ }^{3}$

Acute gouty arthritis is treated with colchicine, nonsteroidal anti-inflammatory drugs (NSAIDs), or glucocorticoids. ULT is indicated in patients with recurrent flares, tophi, urate arthropathy, or renal stones. Xanthine oxidase inhibitors (XOIs), allopurinol and febuxostat, are first-line medications and are considered the gold standard for 
the prevention of recurrent gouty arthritis. Uricosurics, specifically probenecid in the USA, is considered a second-line medication for patients intolerant of, or with contraindications to, XOIs. Uricosurics may also be used in combination with an XOI. Lesinurad is a new uricosuric medication with a novel mechanism of action and has expanded available options for combination therapy. Pegloticase is an expensive intravenous medication that is reserved for gout refractory to conventional therapy.

Despite the availability of multiple guidelines for the management of gout and the introduction of new medication options, gout remains poorly controlled. Factors that may influence persistent symptoms include the high prevalence of cardiometabolic comorbid conditions, poor adherence to ULT, and suboptimal dosing of prophylactic medications. Furthermore, gout is associated with a reduced health-related quality of life (HRQOL), which is possibly related to therapeutic dietary modifications, associated pain, medication concerns, and more frequent health care utilization. ${ }^{4,5}$ This review discusses patient considerations in optimizing the management of gout and the role of combination therapy, focusing on the newest available agent, lesinurad.

\section{Patient profiles}

Men are three times more likely to develop gout than women, with black patients most commonly affected. ${ }^{6}$ The risk of gout increases with age and is especially evident in men older than 30 years and postmenopausal women. Common comorbidities include obesity, hypertension, CKD stage $\geq 2$, diabetes, myocardial infarction, heart failure, and stroke. ${ }^{7}$

A number of dietary factors can influence gout and act as a "trigger" for acute gouty arthritis. Confirmed offenders include excessive consumption of meat, seafood, beer, and liquor. ${ }^{8}$ Dietary modifications alone are likely inadequate to treat most patients diagnosed with gout. However, patients with gout should be advised to avoid or limit consumption of alcohol, particularly beer and spirits, sugar-sweetened beverages, meat, and seafood. In contrast, low-fat dairy products, coffee, and cherries have been negatively associated with gout and consumption can be encouraged. ${ }^{1,2}$ An overall healthy diet, routine exercise, and weight loss, if appropriate, should also be advised due to the strong correlation of gout with concomitant cardiometabolic disease and obesity.

Many patients with gout can be characterized as having poor adherence to treatment. Multiple studies have found that fewer than $50 \%$ of patients are adherent to medication for gout, with some evidence suggesting that nonadherence may be more common in gout than in any other chronic disease. ${ }^{9-11}$
This is particularly true of ULT for the prevention of recurrent gouty arthritis. Lack of education pertaining to the duration of therapy and causes of flares, an awareness that medications may transiently worsen gout symptoms, potential side effect concerns, financial barriers, and an overall disconnect between patients' and providers' views on gout are patient factors that may negatively influence adherence. ${ }^{12,13}$

\section{Clinical end points and patient- related outcomes}

The desired clinical outcomes in gout are to prevent and ultimately eliminate acute gouty arthritis, dissolve tophi, and prevent chronic arthropathy. The 2012 American College of Rheumatology (ACR) and European League against Rheumatism (EULAR) guidelines, along with the $3 \mathrm{e}$ recommendations in rheumatology, endorse a treat-to-target approach to facilitate achievement of clinical outcomes. ${ }^{1,2,14}$ The widely accepted serum urate target level is $<6 \mathrm{mg} / \mathrm{dL}$. However, a lower serum urate target of $<5 \mathrm{mg} / \mathrm{dL}$ is a consideration for patients with severe gout who need fast dissolution of serum urate crystals, characterized by the presence of tophi or chronic arthropathy, or for certain patients with very frequent attacks. Conversely, the 2017 American College of Physicians (ACP) clinical practice guidelines on the management of acute and recurrent gout does not recommend a serum urate target. The ACP guidelines cite that there is insufficient evidence to conclude whether the benefits of a treat-to-target approach outweigh the harms associated with repeated serum urate monitoring and medication escalation. The ACP guidelines propose a "treat-to-avoid symptoms" approach with a goal of avoiding recurrent acute gouty arthritis but without monitoring of serum urate values. ${ }^{3}$

Differences in guideline recommendations regarding the prevention of gouty arthritis have prompted criticism from some experts in the field. ${ }^{15}$ The majority of randomized controlled trials (RCTs) have been developed with a serum urate target as the primary end point, rather than patient-centered clinical outcomes, such as prevention of acute gouty arthritis attacks. This is, in part, due to the short duration of these studies where the incidence of acute gouty arthritis is overall low. In addition, acute gouty arthritis may be temporarily increased following initiation or dose escalation of ULT due to remodeling of tissue urate deposits. For these reasons, reduction in the number of acute gouty arthritis attacks is not an ideal primary end point in studies designed to secure the US Food and Drug Administration (FDA) approval. However, open-label extension studies of allopurinol and febuxostat lasting greater than 1 year demonstrated near 
elimination of gout flares and improved tophus status in the majority of patients. ${ }^{16,17}$ Furthermore, clinical trials evaluating pegloticase, a recombinant pegylated uric acid-specific enzyme that consists of uricase and rapidly and profoundly reduces serum urate in severe gout, provide further evidence that the reduction in serum urate levels to target values correlates with clinical outcomes. ${ }^{18,19}$

Patient-related outcomes associated with gout therapy should be a high priority in clinical trials. There is ample evidence to support an association between the occurrence of gout and a poor HRQOL, but gout-specific patient-related outcomes that contribute to poor HRQOL are not well defined. ${ }^{20}$ One small cohort study identified severe joint pain as the cardinal symptom of gout, accompanied by swelling, redness, heat, sensitivity to touch, and stiffness. ${ }^{21}$ In this study, patients correlated these symptoms with a negative impact on physical functioning, sleep, daily activities, and work. Additional evidence evaluating the benefits of pegloticase in refractory gout further supports that achieving a target serum urate is associated with a positive impact on patientrelated outcomes, improving patient assessment of disease activity, pain, physical function, and HRQOL. ${ }^{22}$ Response to ULT should include both achievement of target serum urate values and patient-reported symptoms, including the impact on daily life and function.

\section{ULT}

The pathophysiology of gout is well understood. When serum urate levels reach a point above the saturation threshold ( $>6.8 \mathrm{mg} / \mathrm{dL}$ at $\mathrm{pH} 7.0$ and temperature of $37^{\circ} \mathrm{C}$, and $>6.0 \mathrm{mg} /$ $\mathrm{dL}$ at $\mathrm{pH} 7.0$ and a temperature of $35^{\circ} \mathrm{C}$ ) monosodium urate (MSU) crystals can form. ${ }^{23,24}$ Reducing serum urate concentrations below the saturation point facilitates the dissolution of MSU crystals and, thus, eventual resolution of gouty arthritis symptoms. Therefore, the use of ULT is the cornerstone of gout management. Recommendations related to the timing of initiation of ULT vary slightly between guidelines and are outlined in Table 1.

The dissolution and mobilization of MSU crystals followed by the initiation or dose titration of ULT can result in increased acute gout symptoms. Acute gout prophylaxis with daily colchicine or low-dose NSAIDs in the presence

Table I Guideline recommendations for ULT

\begin{tabular}{|c|c|c|c|}
\hline $\begin{array}{l}\text { Guideline } \\
\text { (year) }\end{array}$ & Recommendation for ULT & XOI therapies & Combination therapy \\
\hline $\begin{array}{l}A C R^{\prime} \\
(20 \mid 2)\end{array}$ & $\begin{array}{l}\text { Any patient with an established diagnosis of gouty arthritis: } \\
\text { - Tophus or tophi on clinical examination or imaging study } \\
\text { - Frequent attacks of acute gouty arthritis ( } \geq 2 \text { attacks/year) } \\
\text { - CKD stage } 2 \text { or worse } \\
\text { - Past urolithiasis }\end{array}$ & $\begin{array}{l}\text { Allopurinol or febuxostat as first line } \\
\text { Febuxostat can be substituted for } \\
\text { allopurinol or vice versa in the event } \\
\text { of drug intolerance and adverse events, } \\
\text { and such a substitution should be } \\
\text { considered after initial failure of upward } \\
\text { dose titration of one } \mathrm{XOI}\end{array}$ & $\begin{array}{l}\text { The addition of a uricosuric } \\
\text { agent to an XOl drug or vice } \\
\text { versa is an effective option }\end{array}$ \\
\hline $3 e^{14}(2014)$ & Not specifically addressed & $\begin{array}{l}\text { Allopurinol should be used first line. } \\
\text { Low to medium doses of febuxostat } \\
(40-120 \mathrm{mg}) \text { are alternatives in } \\
\text { the presence of intolerance or } \\
\text { nonresponsiveness to allopurinol }\end{array}$ & Not specifically addressed \\
\hline $\begin{array}{l}\text { EULAR }^{2} \\
(2017)\end{array}$ & $\begin{array}{l}\text { Should be considered and discussed with every patient } \\
\text { with a definite diagnosis of gout after the first presentation. } \\
\text { Recommended in all patients with recurrent flares, tophi, urate } \\
\text { arthropathy, and/or renal stones. Initiation is recommended } \\
\text { close to the time of first diagnosis in patients presenting at a } \\
\text { young age ( }<40 \text { years) or with a very high serum urate }(8.0 \mathrm{mg} /\end{array}$ & $\begin{array}{l}\text { Allopurinol as first line in patients with } \\
\text { normal kidney function. If the serum } \\
\text { urate target cannot be reached by an } \\
\text { appropriate dose of allopurinol (or for } \\
\text { intolerance), switch to febuxostat }\end{array}$ & $\begin{array}{l}\text { XOI, particularly allopurinol, } \\
\text { in combination with a } \\
\text { uricosuric is encouraged } \\
\text { when serum urate target is } \\
\text { not achieved or if symptoms } \\
\text { persist on monotherapy }\end{array}$ \\
\hline
\end{tabular}

$\mathrm{ACP}^{3} \quad$ Should not be initiated in most patients after a first gout

(2017) attack or in patients with infrequent attacks.

Clinicians should discuss benefits, harms, costs, and individual

preferences with patients before initiating, including

concomitant prophylaxis, in patients with recurrent gout

attacks

Febuxostat (40 mg/day) and allopurinol (300 mg/day) are equally effective at decreasing serum urate levels
Not specifically addressed

(n)

\author{
a substitution should be \\ Allopurinol should be used first line. \\ (40-120 mg) are alternatives in \\ Allopurinol as first line in patients with \\ in combination with a \\ uricosuric is encouraged \\ not achieved or if symptoms \\ persist on monotherapy
}


of a colchicine contraindication or intolerance should be initiated with ULT and continued during the first 6 months of treatment. $^{2}$

\section{XOls}

Guidelines emphasize that XOI monotherapy, either with allopurinol or febuxostat, is the first-line therapy for the prevention of gout (Table 1). While guidelines do not typically recommend one XOI over the other, allopurinol is more commonly prescribed, likely due to its generic availability and overall cost effectiveness. ${ }^{25}$ In head-to-head and real-life comparisons of allopurinol with febuxostat, attainment of serum urate targets was significantly higher with febuxostat. ${ }^{26,27}$ Importantly, a limitation of these data is suboptimal dosing of allopurinol secondary to the lack of further dose escalation by health care providers. In the head-to-head comparison, $300 \mathrm{mg} /$ day of allopurinol was used, and in the real-life comparison the average allopurinol dose was $184.9 \mathrm{mg} /$ day. With the usual dose of allopurinol of $300 \mathrm{mg} /$ day, the achievement of serum urate targets is less than $50 \%$ in controlled trials. ${ }^{1}$ This is clearly less than desirable. Success rates markedly improve when the allopurinol dose is escalated above this typical dose (much higher than $300 \mathrm{mg} /$ day). ${ }^{28,29}$

The reasons for a conservative allopurinol dosing approach (using no more than $300 \mathrm{mg} /$ day) may be related to product labeling dose restrictions in renal impairment and concern for severe side effects such as allopurinol hypersensitivity syndrome (AHS) which is rare, but potentially life-threatening. While these safety concerns should be considered prior to the initiation of allopurinol, dose escalation beyond $300 \mathrm{mg} /$ day has been proven to be safe..$^{29,30}$ To reduce the risk of AHS, starting doses should not be greater than $100 \mathrm{mg}$ /day for any patient and $50 \mathrm{mg}$ /day for patients with stage 4 or worse CKD. Gradual titration every 2-5 weeks is advised so that doses $>300 \mathrm{mg}$ /day can be used in patients with renal impairment if accompanied by adequate patient education and monitoring. Prior to the initiation of allopurinol, HLA-B*5801 allele testing should be obtained for patients of Han Chinese and Thai descent and should be considered in Koreans and patients with stage 3 or worse CKD. ${ }^{1}$ Allopurinol should be avoided in patients testing positive for this allele due to a higher risk of AHS.

Febuxostat does not require renal dose adjustment and use of this medication does not have the same risk for hypersensitivity reactions as allopurinol. However, caution should be used prior to prescribing following the 2017 FDA Safety Communication. Preliminary results from a safety trial demonstrated increased cardiovascular death when febuxostat was compared to allopurinol. ${ }^{31}$ The trial was designed to compare rates of cardiovascular death, nonfatal myocardial infarction, nonfatal stroke, and unstable angina requiring urgent coronary revascularization between febuxostat 40-80 mg daily and allopurinol 200-600 mg daily over a period up to 60 months. ${ }^{32}$ The primary end point of composite cardiovascular events was not significantly greater in the febuxostat group. However, when the outcomes were evaluated individually, febuxostat showed higher rates of cardiovascular-related death and death from all causes. The FDA will evaluate the final results from the manufacturer when available. ${ }^{31}$ These data do not suggest that complete avoidance or immediate discontinuation of febuxostat is necessary. Rather, health care providers should evaluate patient specific cardiovascular risk versus benefits of febuxostat therapy. When not contraindicated, allopurinol may be preferred.

\section{Uricosurics}

Uricosurics should be used as add-on agents to an XOI when serum urate target is not achieved or if acute gouty arthritis symptoms persist with monotherapy. Uricosurics may be an option as monotherapy in the presence of intolerable adverse effects or contraindications to XOIs. While probenecid is the only available uricosuric approved for gout in the USA and Australia, benzbromarone is an additional agent available in other countries, notably throughout Europe. Benzbromarone is more effective in achieving serum urate target compared to probenecid but may also have a higher risk of hepatotoxicity. Both probenecid and benzbromarone in combination with allopurinol are more effective than allopurinol monotherapy. Overall, high-quality evidence evaluating benzbromarone and probenecid in the reduction in the frequency of gouty arthritis attacks and patient-related outcomes is lacking. ${ }^{33}$ However, the use of these medications is very limited in practice. Other medications such as losartan and fenofibrate have uricosuric effects but are not approved by the FDA for the prevention of gout.

\section{Lesinurad}

Lesinurad was approved by the FDA and European Commission (EC) in 2015. It is available alone as the brand name Zurampic $^{\circledR}$ (Ironwood Pharmaceuticals, Inc., Cambridge, MA, USA) and in combination with allopurinol $200 \mathrm{mg}$ or $300 \mathrm{mg}$ under the brand name Duzallo ${ }^{\circledR}$ (Ironwood Pharmaceuticals, Inc.). Lesinurad has a novel mechanism of action where it decreases serum urate by inhibiting the function 
of transporter proteins (URAT1, OAT4) that reabsorb uric acid in the kidney. URAT1 is responsible for the majority of uric acid reabsorption from the renal tubular lumen, where OAT4 is the transporter involved in diuretic-induced hyperuricemia. Lesinurad is approved by the FDA for use in combination with an XOI for gout; it is not approved for monotherapy.

Combining Lesinurad with Allopurinol Standard of Care in Inadequate Responders (CLEAR) was a two-replicate Phase III trial that provided the requisite data needed for FDA approval. The CLEAR-1 was conducted in the USA, and the CLEAR-2 was conducted in Europe, North America, South Africa, Australia, and New Zealand. ${ }^{34,35}$ In each of these randomized, double-blind trials, patients taking $\geq 300 \mathrm{mg}$ of allopurinol $(\geq 200 \mathrm{mg}$ in patients with moderate renal impairment) with a baseline serum urate of $\geq 6.5 \mathrm{mg} / \mathrm{dL}$ and $\geq 2$ flares in the previous 12 months were randomized to lesinurad $200 \mathrm{mg}$, lesinurad $400 \mathrm{mg}$, or placebo daily. Acute gout prophylaxis was also initiated using daily colchicine or an NSAID in the presence of a colchicine intolerance or contraindication. The primary efficacy end point was the proportion of patients with a serum urate of $<6 \mathrm{mg} / \mathrm{dL}$ by month 6. Key secondary end points included the mean rate of gout flares requiring treatment from the end of month 6 and month 12 and the proportion of patients with tophi at baseline who experienced complete resolution of one or more tophus at month 12 . Safety assessments included treatment-emergent adverse events (TEAEs), laboratory data, physical examination findings, electrocardiogram findings, and vital signs, with renal and cardiovascular safety being the primary focus.

Results of both the CLEAR-1 and CLEAR-2 demonstrated the efficacy of lesinurad. Patients $(n=603$ in CLEAR-1; $n=610$ in CLEAR-2) were predominantly men with a mean age of 51 years, gout duration of 11 years, and baseline serum urate of $6.9 \mathrm{mg} / \mathrm{dL}$, and the majority were taking allopurinol $300 \mathrm{mg}$ daily. Lesinurad at doses of $200 \mathrm{mg}$ or $400 \mathrm{mg}$ added to allopurinol significantly increased the proportion of patients who achieved serum urate target of $<6 \mathrm{mg} / \mathrm{dL}$ when compared to patients receiving allopurinol alone $(54.2 \%, 59.2 \%$, and $27.9 \%, P<0.0001$ : CLEAR-1; $55.4 \%, 66.5 \%$, and $23.3 \%, P<0.0001$ : CLEAR-2). There were no differences in secondary outcomes of gout flares requiring treatment or tophus size, although the short duration of the study may contribute to the lack of positive findings. Lesinurad was generally well tolerated. Lesinurad $200 \mathrm{mg}$ had a comparable safety profile to placebo (Table 2 ), with the exception of renal-related adverse events. Serum creatinine elevations $\geq 1.5 \times$ and $\geq 2 \times$ baseline occurred more commonly in patients taking lesinurad, and this increase was dose dependent (occurring more frequently with lesinurad $400 \mathrm{mg}$ ). The

Table 2 Safety data from lesinurad Phase III clinical trials

\begin{tabular}{|c|c|c|c|c|c|c|c|c|c|}
\hline \multirow{2}{*}{$\begin{array}{l}\text { AE } \\
\text { category }\end{array}$} & \multicolumn{3}{|l|}{ CLEAR- $^{34}$} & \multicolumn{3}{|l|}{ CLEAR-2 $^{35}$} & \multicolumn{3}{|l|}{ CRYSTAL $^{36}$} \\
\hline & $\begin{array}{l}\text { Placebo }+ \\
\text { allopurinol } \\
(n=201)\end{array}$ & $\begin{array}{l}\text { Lesinurad } \\
200 \mathrm{mg}+ \\
\text { allopurinol } \\
(\mathrm{n}=20 \mathrm{I})\end{array}$ & $\begin{array}{l}\text { Lesinurad } \\
400 \mathrm{mg}+ \\
\text { febuxostat } \\
(\mathrm{n}=201)\end{array}$ & $\begin{array}{l}\text { Placebo + } \\
\text { allopurinol } \\
(n=206)\end{array}$ & $\begin{array}{l}\text { Lesinurad } \\
200 \mathrm{mg}+ \\
\text { allopurinol } \\
(n=204)\end{array}$ & $\begin{array}{l}\text { Lesinurad } \\
400 \mathrm{mg}+ \\
\text { febuxostat } \\
(n=200)\end{array}$ & $\begin{array}{l}\text { Placebo }+ \\
\text { febuxostat } \\
(n=109)\end{array}$ & $\begin{array}{l}\text { Lesinurad } \\
200 \text { mg }+ \\
\text { febuxostat } \\
(n=106)\end{array}$ & $\begin{array}{l}\text { Lesinurad } \\
400 \text { mg }+ \\
\text { febuxostat } \\
(n=109)\end{array}$ \\
\hline $\begin{array}{l}\text { Any TEAE } \\
(\%)\end{array}$ & I 38 (68.7) & I 47 (73.I) & $156(77.6)$ & I 46 (70.9) & $152(74.5)$ & 161 (80.5) & $79(72.5)$ & $87(82.1)$ & $90(82.6)$ \\
\hline $\begin{array}{l}\text { Any TEAE } \\
\text { possibly } \\
\text { related to } \\
\text { the study } \\
\text { drug (\%) }\end{array}$ & $19(9.5)$ & $33(16.4)$ & $4 I(20.4)$ & $39(18.9)$ & $40(19.6)$ & $50(25.0)$ & $22(20.2)$ & $25(23.6)$ & $28(25.7)$ \\
\hline $\begin{array}{l}\text { Any } \\
\text { serious } \\
\text { TEAE (\%) }\end{array}$ & II (5.5) & $9(4.5)$ & $16(8.0)$ & $8(3.9)$ & $9(4.4)$ & $19(9.5)$ & $10(9.2)$ & $6(5.7)$ & $9(8.3)$ \\
\hline $\begin{array}{l}\text { Any fatal } \\
\text { TEAE (\%) }\end{array}$ & 0 & I (0.5) & 0 & 0 & 0 & $2(1.0)$ & 0 & I (0.9) & I (0.9) \\
\hline $\begin{array}{l}\text { Renal- } \\
\text { related } \\
\mathrm{AEs}^{\mathrm{a}, \mathrm{b}}(\%)\end{array}$ & $7(3.5)$ & $8(4.0)$ & $20(10.0)$ & $10(4.9)$ & $12(5.9)$ & $30(15)$ & $6(5.5)$ & $9(8.5)$ & II (I0.I) \\
\hline $\begin{array}{l}\text { CV-related } \\
\text { TEAEs (\%) }\end{array}$ & $7(3.5)$ & $9(4.5)$ & $8(4.0)$ & $12(5.9)$ & $8(3.9)$ & $6(3.0)$ & $2(1.8)$ & $6(5.7)$ & $4(3.7)$ \\
\hline
\end{tabular}

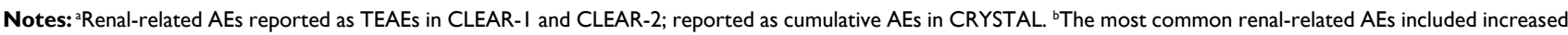
blood creatinine, increased blood urea nitrogen, renal failure, and kidney stones.

Abbreviations: AE, adverse event; CV, cardiovascular; TEAE, treatment-emergent adverse event. 
majority of these cases were transient and reversible with resolution in approximately two-thirds of patients without interruption of lesinurad. Other adverse effects noted across the two studies included upper respiratory tract infections. ${ }^{34,35}$

The Combination Treatment Study in Subjects with Subcutaneous Tophaceous Gout with Lesinurad and Febuxostat (CRYSTAL) trial was a Phase III, multinational trial that investigated the safety and efficacy of lesinurad in combination with febuxostat in patients with tophaceous gout. ${ }^{36}$ Patients with a baseline serum urate of $\geq 8 \mathrm{mg} / \mathrm{dL}$ ( $\geq 6 \mathrm{mg} / \mathrm{dL}$ with ULT) and $\geq 1$ measurable tophus who were treated with febuxostat $80 \mathrm{mg}$ daily for 3 weeks were included in this trial. They were then randomized to lesinurad $200 \mathrm{mg}$, lesinurad $400 \mathrm{mg}$, or placebo daily in addition to febuxostat. Acute gout prophylaxis was initiated concomitantly with febuxostat in the form of daily colchicine or an NSAID in the presence of a colchicine intolerance or contraindication. The primary efficacy end point was the achievement of a serum urate value of $<5 \mathrm{mg} / \mathrm{dL}$ after 6 months. The key secondary end point was the achievement of a complete resolution of $\geq 1$ tophus after 12 months. Safety assessments included TEAEs, laboratory data, physical examination findings, electrocardiogram results, and vital signs, with renal and cardiovascular safety being the primary focus.

Patients in CRYSTAL $(n=324)$ were predominantly white men with a mean age of 54 years, gout duration of 15 years, baseline serum urate of $8.7 \mathrm{mg} / \mathrm{dL}$, and serum urate of $5.3 \mathrm{mg} / \mathrm{dL}$ following 3 weeks of febuxostat. Lesinu$\mathrm{rad} 400 \mathrm{mg}$ added to febuxostat significantly increased the proportion of patients who achieved serum urate target of $<5 \mathrm{mg} / \mathrm{dL}$ after 6 months when compared to patients receiving febuxostat alone $(76.1 \%, 46.8 \%$, respectively; $P<0.0001)$. Lesinurad $200 \mathrm{mg}$ added to febuxostat did not significantly increase the proportion of patients achieving serum urate target after 6 months but did at all other time points. There was no difference between the number of patients with complete tophus resolution. However, lesinurad $200 \mathrm{mg}$ and $400 \mathrm{mg}$ added to febuxostat did significantly reduce the total target tophi area when compared to febuxostat alone $(50.1 \%$, $52.9 \%, 28.3 \%$, respectively; $P<0.05)$.

Lesinurad was generally well tolerated in the CRYSTAL study (Table 2). The proportion of patients with TEAEs throughout the study was $72.5 \%$ in the febuxostat alone group, $82.1 \%$ with lesinurad $200 \mathrm{mg}$ plus febuxostat and $82.6 \%$ with lesinurad $400 \mathrm{mg}$ plus febuxostat. Serum creatinine elevations $\geq 1.5 \times$ baseline occurred in $2.8 \%(n=3), 4.7 \%$ $(n=5)$, and $10.1 \%(n=11)$ of patients in the febuxostat alone, lesinurad $200 \mathrm{mg}$ plus febuxostat, and lesinurad $400 \mathrm{mg}$ plus febuxostat groups, respectively. Of these, $100 \%, 60 \%$, and $85.7 \%$ resolved without interruption of the study drug. Serum creatinine elevations $\geq 2 \times$ baseline occurred in $0 \%$, $2.8 \%(n=3)$, and $5.5 \%(n=6)$ of the respective groups. Only one patient in each of the lesinurad plus febuxostat groups had unresolved serum creatinine elevations at the last study assessment. Other individual adverse effects noted were nasopharyngitis, hypertension, headache, extremity pain, and back pain. ${ }^{36}$

Lesinurad should never be used as monotherapy in patients with gout. In one study comparing lesinurad $400 \mathrm{mg}$ daily to placebo, there was a high incidence of serum creatinine elevations and renal-related adverse events, including kidney stones, renal failure, and acute renal failure. ${ }^{37}$ When lesinurad is used in the absence of an XOI, the rate of urinary uric acid excretion is higher and has the potential to induce uric acid microcrystallization in the renal tubules, resulting in renal-related adverse effects. Thus, lesinurad is not approved by the FDA for use as monotherapy.

Based on the findings of these studies, lesinurad is only available in the $200 \mathrm{mg}$ dose and has a black-boxed warning that provides safety information related to acute renal failure. ${ }^{38}$ With approval, the FDA and EC mandated postmarketing studies to more fully evaluate renal and cardiovascular safety of this novel agent.

\section{Lesinurad: role in therapy}

Currently, no studies have evaluated lesinurad's effect on patient-related outcomes such as pain, physical function, and impact on daily life. However, evidence with other available medications has correlated achievement of serum urate targets with improvements in clinical outcomes and patient-related outcomes. Presumably, this should be true with lesinurad. However, because lesinurad has a unique mechanism of action, this cannot be assumed. Because lesinurad is still a relatively new therapy, to date, uptake by health care professionals has not been widespread. According to the manufacturer's quarterly reports, a total of only 4,466 prescriptions for lesinurad were filled in 2017 through the third quarter. ${ }^{39-41}$ However, interest in using allopurinol with lesinurad may increase in the wake of the febuxostat FDA Safety Communication and the new availability of a fixeddose allopurinol/lesinurad combination product in 2017.

While this first step to providing maximum ULT is optimizing the dose of allopurinol up to $800 \mathrm{mg}$ daily to achieve maximum efficacy, lesinurad may be of benefit for selected patients. Patients who do not achieve individualized serum urate target of $<6 \mathrm{mg} / \mathrm{dL}$ or $<5 \mathrm{mg} / \mathrm{dL}$ or those with 
continued severe gout symptoms (tophi, chronic arthropathy, frequent attacks) on a maximally tolerated XOI would be ideal candidates for lesinurad treatment. Because lesinurad can be used in patients with a creatinine clearance as low as $45 \mathrm{~mL} / \mathrm{min}$, it may be particularly useful in patients with moderate CKD where dose titration of allopurinol may be limited. Finally, there is some evidence that dyslipidemia, abdominal obesity, and alcohol consumption are factors that may be associated with a poor response to ULT. ${ }^{42}$ The presence of these comorbidities may represent another patient population where lesinurad could be considered.

Ultimately, the use of lesinurad should be determined by provider and patient preferences as it relates to risks, benefits, and cost. Patients who are prescribed lesinurad should have a baseline renal function assessment followed by periodic monitoring. Patients prescribed lesinurad should be informed of known side effects and instructed to maintain adequate hydration (clinical trials demonstrating efficacy with lesinurad encouraged a $2 \mathrm{~L}$ intake of fluid daily).

\section{Conclusion}

Despite a well-understood pathophysiologic mechanism of disease, generally consistent guideline recommendations, and effective treatment options, gout remains a poorly controlled disease. In light of concerning cardiovascular findings with febuxostat, health care providers should optimize the doses of allopurinol to achieve serum urate targets. If additional therapy is warranted, lesinurad is a new medication that, when combined with an XOI, is effective in reaching serum urate targets. Clinical efficacy and improvements in patientrelated outcomes have yet to be demonstrated with the use of lesinurad. Clinical studies have not been designed to assess these outcomes as primary end points with lesinurad therapy. Future studies should be of longer duration, include clinical efficacy primary outcomes such as episodes of recurrent gouty arthritis requiring treatment, and ensure that an optimized dose of allopurinol $>300 \mathrm{mg}$ daily is utilized.

\section{Disclosure}

The authors report no conflicts of interest in this work.

\section{References}

1. Khanna D, Fitzgerald JD, Khanna PP, et al. 2012 American College of Rheumatology guidelines for management of gout. Part 1: systematic nonpharmacologic and pharmacologic therapeutic approaches to hyperuricemia. Arthritis Care Res (Hoboken). 2012;64(10):1431-1446.

2. Richette P, Doherty M, Pascual E, et al. 2016 updated EULAR evidencebased recommendations for the management of gout. Ann Rheum Dis. 2017;76(1):29-42.
3. Qaseem A, Harris RP, Forciea MA; Clinical Guidelines Committee of the American College of Physicians. Management of acute and recurrent gout: a clinical practice guideline from the American College of Physicians. Ann Intern Med. 2017;166(1):58-68.

4. Singh JA, Strand V. Gout is associated with more comorbidities, poorer health-related quality of life and higher healthcare utilisation in US veterans. Ann Rheum Dis. 2008;67(9):1310-1316.

5. Singh JA. Challenges faced by patients in gout treatment: a qualitative study. J Clin Rheumatol. 2014;20(3):172-174.

6. Wilson L, Saseen JJ. Gouty arthritis: a review of acute management and prevention. Pharmacotherapy. 2016;36(8):906-922.

7. Zhu Y, Pandya BJ, Choi HK. Comorbidities of gout and hyperuricemia in the US general population: NHANES 2007-2008. Am J Med. 2012;125(7):679-687.

8. Choi HK. A prescription for lifestyle change in patients with hyperuricemia and gout. Curr Opin Rheumatol. 2010;22(2):165-172.

9. Scheepers LEJM, van Onna M, Stehouwer CDA, Singh JA, Arts ICW, Boonen A. Medication adherence among patients with gout: a systematic review and meta-analysis. Semin Arthritis Rheum. 2018;47(5):689-702.

10. De Vera MA, Marcotte G, Rai S, Galo JS, Bhole V. Medication adherence in gout: a systematic review. Arthritis Care Res (Hoboken). 2014;66(10):1551-1559.

11. Briesacher BA, Andrade SE, Fouayzi H, Chan KA. Comparison of drug adherence rates among patients with seven different medical conditions. Pharmacotherapy. 2008;28(4):437-443.

12. Harrold LR, Mazor KM, Velten S, Ockene IS, Yood RA. Patients and providers view gout differently: a qualitative study. Chronic Illn. 2010;6(4):263-271.

13. Nasser-Ghodsi N, Harrold LR. Overcoming adherence issues and other barriers to optimal care in gout. Curr Opin Rheumatol. 2015;27(2): 134-138.

14. Sivera F, Andrés M, Carmona L, et al. Multinational evidence-based recommendations for the diagnosis and management of gout: integrating systematic literature review and expert opinion of a broad panel of rheumatologists in the 3e initiative. Ann Rheum Dis. 2014;73(2):328-335.

15. Dalbeth N, Bardin T, Doherty M, et al. Discordant American College of Physicians and international rheumatology guidelines for gout management: consensus statement of the Gout, Hyperuricemia and Crystal-Associated Disease Network (G-CAN). Nat Rev Rheumatol. 2017;13(9):561-568.

16. Becker MA, Schumacher HR, MacDonald PA, Lloyd E, Lademacher C. Clinical efficacy and safety of successful longterm urate lowering with febuxostat or allopurinol in subjects with gout. $J$ Rheumatol. 2009;36(6):1273-1282.

17. Schumacher HR, Becker MA, Lloyd E, MacDonald PA, Lademacher C. Febuxostat in the treatment of gout: 5-yr findings of the FOCUS efficacy and safety study. Rheumatology. 2008;48(2):188-194.

18. Sundy JS, Baraf HSB, Yood RA, et al. Efficacy and tolerability of pegloticase for the treatment of chronic gout in patients refractory to conventional treatment: two randomized controlled trials. JAMA. 2011;306(7):711-720.

19. Becker MA, Baraf HSB, Yood RA, et al. Long-term safety of pegloticase in chronic gout refractory to conventional treatment. Ann Rheum Dis. 2013;72(9):1469-1474.

20. Chandratre P, Roddy E, Clarson L, Richardson J, Hider SL, Mallen CD. Health-related quality of life in gout: a systematic review. Rheumatology (Oxford). 2013;52(11):2031-2040.

21. Tatlock S, Rüdell K, Panter C, et al. What outcomes are important for gout patients? In-depth qualitative research into the gout patient experience to determine optimal endpoints for evaluating therapeutic interventions. Patient. 2017;10(1):65-79.

22. Strand V, Khanna D, SIingh JA, Forsythe A, Edwards NL. Improved health-related quality of life and physical function in patients with refractory chronic gout following treatment with pegloticase: evidence from phase III randomized controlled trials. $J$ Rheumatol. 2012;39(7):1450-1457. 
23. Loeb JN. The influence of temperature on the solubility of monosodium urate. Arthritis Rheum. 1972;15(2):189-192.

24. Chhana A, Lee G, Dalbeth N. Factors influencing the crystallization of monosodium urate: a systematic literature review. BMC Musculoskelet Disord. 2015;16(1):296.

25. Guerra T [webpage on the Internet]. The Top 200 Drugs of 2017? 2017. Available from: http://www.pharmacytimes.com/contributor/ tony-guerra-pharmd/2017/03/the-top-200-drugs-of-2017. Accessed December 16, 2017.

26. Hatoum H, Khanna D, Lin S, Akhras KS, Shiozawa A, Khanna P. Achieving serum urate goal: a comparative effectiveness study between allopurinol and febuxostat. Postgrad Med. 2014;126(2):65-75.

27. Becker MA, Schumacher HR, Wortmann RL, et al. Febuxostat compared with allopurinol in patients with hyperuricemia and gout. NEngl JMed. 2005;353(23):2450-2461.

28. Reinders MK, Haagsma C, Jansen TL, et al. A randomised controlled trial on the efficacy and tolerability with dose escalation of allopurinol $300-600 \mathrm{mg} /$ day versus benzbromarone $100-200 \mathrm{mg}$ /day in patients with gout. Ann Rheum Dis. 2009;68(6):892-897.

29. Stamp LK, O'Donnell JL, Zhang M, et al. Using allopurinol above the dose based on creatinine clearance is effective and safe in patients with chronic gout, including those with renal impairment. Arthritis Rheum. 2011;63(2):412-421.

30. Stamp LK, Chapman PT, Barclay M, et al. Allopurinol dose escalation to achieve serum urate below $6 \mathrm{mg} / \mathrm{dL}$ : an open-label extension study. Ann Rheum Dis. 2017;76(12):2065-2070.

31. Drug Safety and Availability [webpage on the Internet]. FDA Drug Safety Communication: FDA to Evaluate Increased Risk of HeartRelated Death and Death from All Causes with the Gout Medicine Febuxostat (Uloric). 2017. Available from: https://www.fda.gov/Drugs/ DrugSafety/ucm584702.htm. Accessed December 16, 2017.

32. White WB, Chohan S, Dabholkar A, Hunt B, Jackson R. Cardiovascular safety of febuxostat and allopurinol in patients with gout and cardiovascular comorbidities. Am Heart J. 2012;164(1):14-20.

33. Kydd AS, Seth R, Buchbinder R, Edwards CJ, Bombardier C. Uricosuric medications for chronic gout. Kydd AS, ed. Cochrane Database Syst Rev. 2014;11:CD010457.
34. Saag KG, Fitz-Patrick D, Kopicko J, et al. Lesinurad combined with allopurinol: a randomized, double-blind, placebo-controlled study in gout patients with an inadequate response to standard-of-care allopurinol (a US-Based study). Arthritis Rheumatol. 2017;69(1):203-212.

35. Bardin T, Keenan RT, Khanna PP, et al. Lesinurad in combination with allopurinol: a randomised, double-blind, placebo-controlled study in patients with gout with inadequate response to standard of care (the multinational CLEAR 2 study). Ann Rheum Dis. 2017;76(5): 811-820.

36. Dalbeth N, Jones G, Terkeltaub R, et al. Lesinurad, a selective uric acid reabsorption inhibitor, in combination with febuxostat in patients with tophaceous gout findings of a phase III clinical trial. Arthritis Rheumatol. 2017;69(9):1903-1913.

37. Tausche AK, Alten R, Dalbeth N, et al. Lesinurad monotherapy in gout patients intolerant to a xanthine oxidase inhibitor: a 6 month phase 3 clinical trial and extension study. Rheumatology (Oxford). 2017;56(12): 2170-2178.

38. Zurampic (lesinurad) [package insert]. Wilmington, DE: AstraZeneca Pharmaceutical LP; 2015.

39. Ironwood Pharmaceuticals [webpage on the Internet]. Ironwood Pharmaceuticals Provides First Quarter 2017 Investor Update. 2017. Available from: http://investor.ironwoodpharma.com/releasedetail. cfm?ReleaseID=1025239. Accessed December 17, 2017.

40. Ironwood Pharmaceuticals [webpage on the Internet]. Ironwood Pharmaceuticals Provides Second Quarter 2017 Investor Update (NASDAQ:IRWD). 2017. Available from: http://investor.ironwoodpharma.com/releasedetail.cfm?ReleaseID=1035981. Accessed December 17, 2017.

41. Ironwood Pharmaceuticals [webpage on the Internet]. Ironwood Pharmaceuticals Provides Third Quarter 2017 Investor Update. 2017. Available from: http://investor.ironwoodpharma.com/releasedetail. $\mathrm{cfm}$ ?ReleaseID=1046754. Accessed December 17, 2017.

42. Latourte A, Bardin T, Clerson P, Ea H-K, Flipo R-M, Richette P. Dyslipidemia, alcohol consumption and obesity are the main factors associated with poor control of urate levels in patients receiving urate-lowering therapy. Arthritis Care Res (Hoboken). Epub 2017 Aug 22.
Patient Related Outcome Measures

\section{Publish your work in this journal}

Patient Related Outcome Measures is an international, peer-reviewed, open access journal focusing on treatment outcomes specifically relevant to patients. All aspects of patient care are addressed within the journal and practitioners from all disciplines are invited to submit their work as well as healthcare researchers and patient support groups.

\section{Dovepress}

The journal is included in PubMed. The manuscript management system is completely online and includes a very quick and fair peer-review system. Visit http://www.dovepress.com/testimonials.php to read real quotes from published authors. 